\title{
Nível de conhecimento sobre depressão de agentes comunitários de saúde de Belém, Pará
}

\author{
Knowledge level about depression of community health agents of Belém, Pará
}

Nivel de conocimiento sobre la depresión de los agentes de salud comunitaria de Belém, Pará

Anna Karynna Barbosa Gomes ${ }^{1 *}$, Amanda Leal Porto Brito Santos ${ }^{1}$, Ananda Maciel Paiva ${ }^{1}$, Eduardo André Louzeiro Lama ${ }^{1}$, Felipy de França Oliveira ${ }^{1}$, Nathália Jucá de Azevedo Picanço ${ }^{1}$, Allen Washington Duarte Magalhães ${ }^{1}$, Brenda Melo Sampaio ${ }^{1}$, Elton Cardoso Pinheiro ${ }^{1}$, Osias Pimenta Nunes Filho'.

\section{RESUMO}

Objetivo: Analisar o nível de conhecimento de Agentes Comunitários da Saúde (ACS's) de uma Estratégia de Saúde da Família (ESF) da cidade de Belém - PA sobre a depressão. Metodologia: Estudo transversal, descritivo e intervencionista, aprovado pelo Comitê de Ética em Pesquisa (CEP) que avaliou o conhecimento de ACS's sobre depressão por meio de um questionário sóciodemográfico e um para avaliar o nível de conhecimentos sobre a patologia. Resultados: Participaram 11 ACS's, sendo $63,63 \%$ do sexo feminino, $54,55 \%$ com idade entre $30-39$ anos, $54,55 \%$ possuíam ensino superior incompleto, $73 \%$ possuíam formação para ACS, $64 \%$ não conheciam nenhum método de prevenção da depressão $(p<0,0051)$, e $55 \%$ não conheciam o tratamento, mas todos consideraram válido receber treinamento em transtornos mentais $(p<0,0001)$. Após a intervenção, 100\% dos agentes conheciam os sinais e sintomas de depressão $(p<0,0001)$, relatando que existem métodos preventivos $(p<0,0051)$ e que sabiam que o tratamento era realizado com medicamentos e abordagem psicoterapéutica $(p<0,0001)$. Conclusão: Os ACS's pesquisados conhecem a importância do assunto (depressão) e que intervenção precoce no paciente pode ser importante no curso da doença, contudo desconhecem o curso clínico da doença, sua prevenção, e o fato de ser uma condição incapacitante.

Palavras-chave: Agentes comunitários de saúde, Conhecimento, Depressão.

\begin{abstract}
Objective: To analyze the knowledge level of Community Health Agents (CHA's) of a Family Health Strategy (FHS) of the city of Belém - PA about depression. Methodology: Cross-sectional, descriptive and interventional study, approved by the Research Ethics Committee (CEP), which evaluated the knowledge of CHA's about depression through a sociodemographic questionnaire and one to assess the level of knowledge about the disease. Results: Eleven CHA's participated, being $63.63 \%$ female, $54.55 \%$ aged $30-39$ years, $54.55 \%$ had incomplete higher education, $73 \%$ had training for $\mathrm{CHA}, 64 \%$ did not know any method to prevent depression $(p<0.0051)$ and $55 \%$ did not know the treatment, but all considered valid to receive training in mental disorders $(p<0.0001)$. After the intervention, $100 \%$ of the agents knew the signs and symptoms of depression $(p<0.0001)$, reporting that there are preventive methods $(p<0.0051)$ and that they knew that the treatment was performed with medication and psychotherapeutic approach $(p<0.0001)$.Conclusion: The ACSs surveyed know the importance of the subject (depression) and that early intervention in the patient may be important in the course of the disease, but do not know the clinical course of the disease, its prevention, and the fact that it is a disabling condition.
\end{abstract}

Keywords: Community health works, Knowledge, Depression.

${ }^{1}$ Centro Universitário Metropolitano da Amazonia (UNIFAMAZ), Belém - Pará.

*E-mail: annakarynnabgomes@gmail.com 


\section{RESUMEN}

Objetivo: Analizar el nivel de conocimiento de los Agentes Comunitarios de Salud (ACS's) de una Estrategia de Salud de la Familia (ESF) de la ciudad de Belém - PA sobre la depresión. Metodología: Estudio transversal, descriptivo e intervencionista, aprobado por el Comité de Ética en Investigación (CEP), que evaluó el conocimiento de los CHA sobre la depresión a través de un cuestionario sociodemográfico y uno para evaluar el nivel de conocimiento sobre la enfermedad. Resultados: Participaron once ACS's, siendo 63.63\% mujeres, $54.55 \%$ entre 30 y 39 años, $54.55 \%$ tenían educación superior incompleta, $73 \%$ tenían capacitación para CHA, 64\% no conocían ningún método para prevenir depresión $(p<0.0051)$ y el $55 \%$ no conocía el tratamiento, pero todos considerados válidos para recibir entrenamiento en trastornos mentales $(p<0.0001)$. Después de la intervención, el $100 \%$ de los agentes conocía los signos y síntomas de la depresión $(p<0.0001)$, informando que existen métodos preventivos $(p<0.0051)$ y que sabían que el tratamiento se realizó con medicamentos y enfoque psicoterapéutico $(p<0,0001)$. Conclusión: los ACS encuestados conocen la importancia del sujeto (depresión) y que la intervención temprana en el paciente puede ser importante en el curso de la enfermedad, pero no conoce el curso clínico de la enfermedad, su prevención y el hecho de que es una condición incapacitante.

Palabras clave: Agentes comunitarios de salud, Conocimiento, Depresión.

\section{INTRODUÇÃO}

A depressão é o distúrbio psiquiátrico mais comum na população mundial e o transtorno mental mais frequente nos cuidados primários de saúde. É uma doença grave, com imenso sofrimento mental e tendência a recidiva caracterizada por mau humor, sensação de tristeza, melancolia e vazio, e geralmente começa após um evento negativo da vida, sendo mais comumente reconhecida entre pacientes em um atendimento primário de saúde.

Também é caracterizada por perda de interesse e prazer nas atividades cotidianas, perda de autoconfiança e respeito próprio, culpa, distúrbios do sono, dor de cabeça, aumento ou diminuição do apetite, diminuição da concentração, cansaço e depreciação (RAIC M, 2017). A depressão tem sido reportada como a maior causa única para a incapacidade global $(7,5 \%$ em 2015) e o maior fator contribuinte para a ocorrência de suicídios (aproximadamente 800.000 casos anualmente) (PILANIA M, et al., 2019).

Segundo a World Health Organization (WHO, 2017), mundialmente, estima-se que cerca de 322 milhões de pessoas sofram de depressão, equivalente a $4,4 \%$ da população mundial. A depressão é mais comum entre mulheres $(5,1 \%)$ do que homens $(3,6 \%)$.

A prevalência varia de acordo com a região, com uma taxa de 2,6\% entre os homens na região do Pacífico Ocidental e 5,9\% entre as mulheres na região africana.

As taxas de prevalência variam de acordo com a idade, atingindo o pico na idade adulta (acima de $7,5 \%$ entre as mulheres de 55 a 74 anos e acima de 5,5\% entre os homens). A depressão também ocorre em crianças e adolescentes com menos de 15 anos, mas em um nível mais baixo do que as faixas etárias mais velhas.

De acordo com o Centro Cultural do Ministério da Saúde, houve no final da década de 70 o início da Reforma Psiquiátrica Brasileira, a qual teve como principal objetivo a reinserção do portador de transtornos mentais na sociedade e o desenvolvimento de novas estratégias de atendimento a essa população, investindo na participação da família como núcleo fundamental (CENTRO CULTURAL DO MINISTÉRIO DA SAÚDE, 2006; SILVA JUNIOR RF, et al., 2016; BRASIL, 2012).

Outro objetivo da Reforma foi a desinstitucionalização de pacientes, levando os atendimentos para os centros especializados como, por exemplo, o Centro de Atenção Psicossocial (CAPS). Este último tem substituído a internação de longos períodos por um tratamento que envolve os familiares no atendimento com a atenção necessária, melhorando a recuperação e reintegração social do indivíduo com transtorno mental, conforme revisão de literatura fundamentada na análise do papel do Agente Comunitário de Saúde (ACS) acerca de portadores de transtorno mental na Estratégia de Saúde da Família (ESF), em 2016 (SILVA JUNIOR RF, et al., 2016). 
Segundo o Departamento de Atenção Básica (DAB), a ESF é composta por equipe multiprofissional que possui, no mínimo, médico generalista ou especialista em saúde da família ou médico de família e comunidade, enfermeiro generalista ou especialista em saúde da família, auxiliar ou técnico de enfermagem e ACS's (SILVA JUNIOR RF, et al., 2016; BRASIL, 2012).

O ACS é responsável por fazer a coleta de informações sobre as necessidades de saúde da comunidade, realizar busca ativa e encaminhamento de usuários com problemas à Unidade Básica de Saúde (UBS), além de atuarem como elo entre a população que está sob sua responsabilidade e a equipe de saúde, uma vez que os usuários se identificam com este profissional por residirem na mesma localidade.

Dessa maneira, o ACS é um profissional estratégico na reorientação da Atenção Primária à Saúde (APS) por criar vínculos efetivos com a comunidade em seu território. Assim eles atuam com saber científico e o saber popular facilitando a implantação de estratégias importantes ao atendimento na saúde mental (SILVA JUNIOR RF, et al., 2016).

Dessa forma, por ser uma condição relativamente frequente, de curso crônico e recorrente, a depressão está associada muitas vezes com incapacitação funcional e comprometimento da saúde física, limitando as atividades e o bem-estar dos pacientes, além de fazer com que estes utilizem mais os de serviços de saúde (BRASIL, 2006).

Sendo essa um transtorno mental, a ESF tem, teoricamente, maior potencialidade para lidar com esses problemas relacionados à saúde mental, apesar da carência de capacitação dos profissionais desta estratégia para lidar com tais questões, logo faz-se necessário, em especial, a capacitação do ACS pelo fato de possuir maior proximidade com os usuários (FLICK MP, et al., 2009; VITTA FCF, et al., 2012; KODA MY e FERNANDES MIA, 2007; HARADA OL e SOARES MH, 2010). Portanto, percebe-se então que diante desse novo cenário que a política nacional da saúde mental trouxe, o ACS é membro fundamental no processo de consolidação da reforma psiquiátrica, mas que precisa de mais capacitações efetivas para lidar com esses pacientes (KODA MY e FERNANDES MIA, 2007).

Nesse contexto, o objetivo deste estudo foi analisar o nível de conhecimento de Agentes Comunitários da Saúde (ACS's) de uma Estratégia de Saúde da Família (ESF) da cidade de Belém - PA sobre a depressão e capacitá-los para identificar os principais sinais e sintomas da patologia.

\section{MÉTODOS}

Tratou-se de um estudo transversal, descritivo e intervencionista, sem financiamento externo, que identificou o nível de conhecimento dos ACS's de uma ESF da cidade de Belém - PA a respeito de diversos aspectos relacionados à depressão, proporcionando a eles capacitação acerca do referido transtorno mental, por meio de palestras socioeducativas e atividades lúdicas. O projeto foi aprovado pelo Comitê de Ética em Pesquisa sob CAAE no 82845918.0.0000.5701.

No primeiro momento, foi aplicado um questionário semiestruturado e auto respondido para avaliação do conhecimento dos ACS's acerca da depressão. Após identificar as lacunas no conhecimento dos participantes sobre o transtorno mental, foram realizadas intervenções com a finalidade de erradicar dúvidas sobre o tema.

O segundo momento correspondeu às intervenções: foram realizadas palestras com o auxílio de um especialista da área abordando a epidemiologia, fatores de risco, sinais e sintomas, diagnóstico, tipos de tratamento e complicações da depressão.

O terceiro momento ocorreu após as intervenções, no qual foi repassado um questionário produzido pelo especialista da área que ministrou a palestra contendo perguntas básicas sobre os assuntos abordados na mesma com o objetivo de verificar a efetividade das intervenções realizadas.

Os dados de interesse foram obtidos com o auxílio da aplicação de um questionário adaptado de um artigo publicado na Revista Eletrônica Saúde Mental Álcool e Drogas (HARADA OL e SOARES MH, 2010), contendo informações a respeito dos dados pessoais como idade, sexo e nível de escolaridade, além de perguntas abertas, elaboradas com o propósito de avaliar o conhecimento dos ACS's sobre a depressão. 
O estudo e tabulação estatística dos dados foram feitos usando softwares específicos (Microsoft Excel, Biostat 5.0), utilizando-se também de estatística descritiva e do teste estatístico Qui-Quadrado de Pearson. As variáveis contínuas foram expressas sob a forma de média com ajuste por desvio padrão e as categóricas foram expressas sob a forma de frequência absoluta e relativa. Os resultados obtidos foram expressos sob a forma de gráficos e/ou tabelas.

\section{RESULTADOS}

A Tabela 1 mostra o perfil sociodemográfico dos ACS's participantes da pesquisa. A maioria pertencia ao sexo feminino (63.63\%). A idade dos ACS' variou de 20 a 59 anos, onde $54,55 \%$ apresentavam idade entre 30 e 39 anos. Apenas $18,18 \%$ dos participantes possuíam ensino superior completo, embora a maioria $(54,55 \%)$ tenha ensino superior incompleto.

Tabela 1 - Aspectos sociodemográficos dos Agentes Comunitários de Saúde participantes da pesquisa ( $\mathrm{N}=11)$. Belém, Pará, 2019.

\begin{tabular}{ccc}
\hline Variáveis & N & $\%$ \\
\hline Sexo & 4 & \\
\hline Masculino & 7 & 36,36 \\
Feminino & & 63,63 \\
\hline Faixa etária & 1 & \\
\hline 20 a 29 anos & 6 & 9,09 \\
30 a 39 anos & 1 & 54,55 \\
40 a 49 anos & 3 & 9,09 \\
50 a 59 anos & & 27,27 \\
\hline Escolaridade & 3 & 27,27 \\
\hline Médio completo & 6 & 54,55 \\
Superior incompleto & 2 & 18,18 \\
Superior completo & 11 & 100,0 \\
\hline Total &
\end{tabular}

Fonte: Gomes AKB, et al., 2019.

A Tabela 2 apresenta os dados relativos ao tempo de trabalho e formação para atuar na profissão de ACS, além de capacitação em saúde mental. Em relação ao tempo de trabalho na ESF, 100\% dos participantes atuavam há mais de 24 meses, apresentando, desta forma, experiência com o modelo de Atenção Básica. Entretanto, desta totalidade, somente $73 \%$ possuíam formação para atuar como ACS, o que representa um percentual de $27 \%$ dos ACS's atuando por um período superior a 24 meses na Atenção Básica sem formação inicial para atuar na profissão.

Outro indicador considerado na pesquisa foi sobre o treinamento prévio em saúde mental. Quando questionados sobre a realização de capacitações prévias em saúde mental em anos anteriores ou neste ano, 91\% dos pesquisados disseram ter realizado capacitação anteriormente.

É ainda interessante observar que $100 \%$ dos participantes consideravam válido receber treinamento sobre transtorno mental e depressão, mostrando interesse dos ACS's sobre o tema, bem como na importância deste no contexto do modelo de atenção em saúde na ESF desta comunidade. 
Tabela 2 - Dados relativos ao tempo de trabalho e formação para a profissão e capacitação em saúde mental dos Agentes Comunitários de Saúde participantes da pesquisa ( $N=11)$. Belém, Pará, 2019.

Questões N N $\quad \%$

\section{Tempo de trabalho na ESF}

\begin{tabular}{ccc}
\hline Até 6 meses & 0 & 0,0 \\
De 6 a 18 meses & 0 & 0,0 \\
De 18 a 24 meses & 0 & 0,0 \\
Mais de 24 meses & 11 & 100,0 \\
\hline
\end{tabular}

Formação para ACS

\begin{tabular}{llll}
\hline Sim & 8 & 73,0 \\
Não & 3 & 27,0 \\
\hline
\end{tabular}

\section{Capacitação sobre saúde mental}

\begin{tabular}{ccc}
\hline Este ano & 0 & 0,0 \\
Anos anteriores & 10 & 91,0 \\
Nunca recebi & 1 & 9,0 \\
\hline
\end{tabular}

\section{É importante receber treinamento sobre a depressão}

\begin{tabular}{cccc}
\hline Sim & 11 & 100,0 \\
Não & 0 & 0,0 \\
\hline Total & 11 & 100,0
\end{tabular}

Legenda: ESF: Estratégia Saúde da Família, ACS: Agente Comunitário de Saúde.

Fonte: Gomes AKB, et al., 2019.

A Tabela 3 apresenta os dados referentes às respostas aos questionários antes e após as intervenções. Ao responderem sobre o reconhecimento dos principais sintomas do transtorno, $82 \%$ afirmaram saber identificá-los $(p<0,0001)$. Ao comparar com as respostas do questionário 2 aplicado após as palestras, os ACS's responderam que os principais sintomas da depressão eram humor deprimido (43\%) e perda de interesse $(43 \%)(p=0,0002)$.

Quando questionados sobre os métodos de depressão da doença, antes da intervenção, apenas $36 \%$ responderam que conheciam ( $p=0,0051)$, no entanto, após a intervenção, esse percentual aumentou para $71 \%(p=0,0051)$.

Em relação ao tratamento dos pacientes com depressão, 55\% não sabiam como funcionava o atendimento dessas pessoas antes das palestras $(p=0,3173)$, porém, após a intervenção, $100 \%$ afirmaram que 0 tratamento é realizado com intervenção psicológica associada ao uso de medicamentos $(p<0,0001)$.

Sobre os níveis de referência em saúde mental, 55\% afirmaram não ter conhecimento sobre a existência de um centro de referência para tratamento da depressão e demais transtornos mentais ( $p=0,3173$ ); após as palestras, $100 \%$ dos ACS's tiveram respostas positivas $(p<0,0001)$. 
Tabela 3 - Conhecimento dos Agentes Comunitários de Saúde sobre a depressão antes e após a intervenção (N=11). Belém, Pará, 2019.

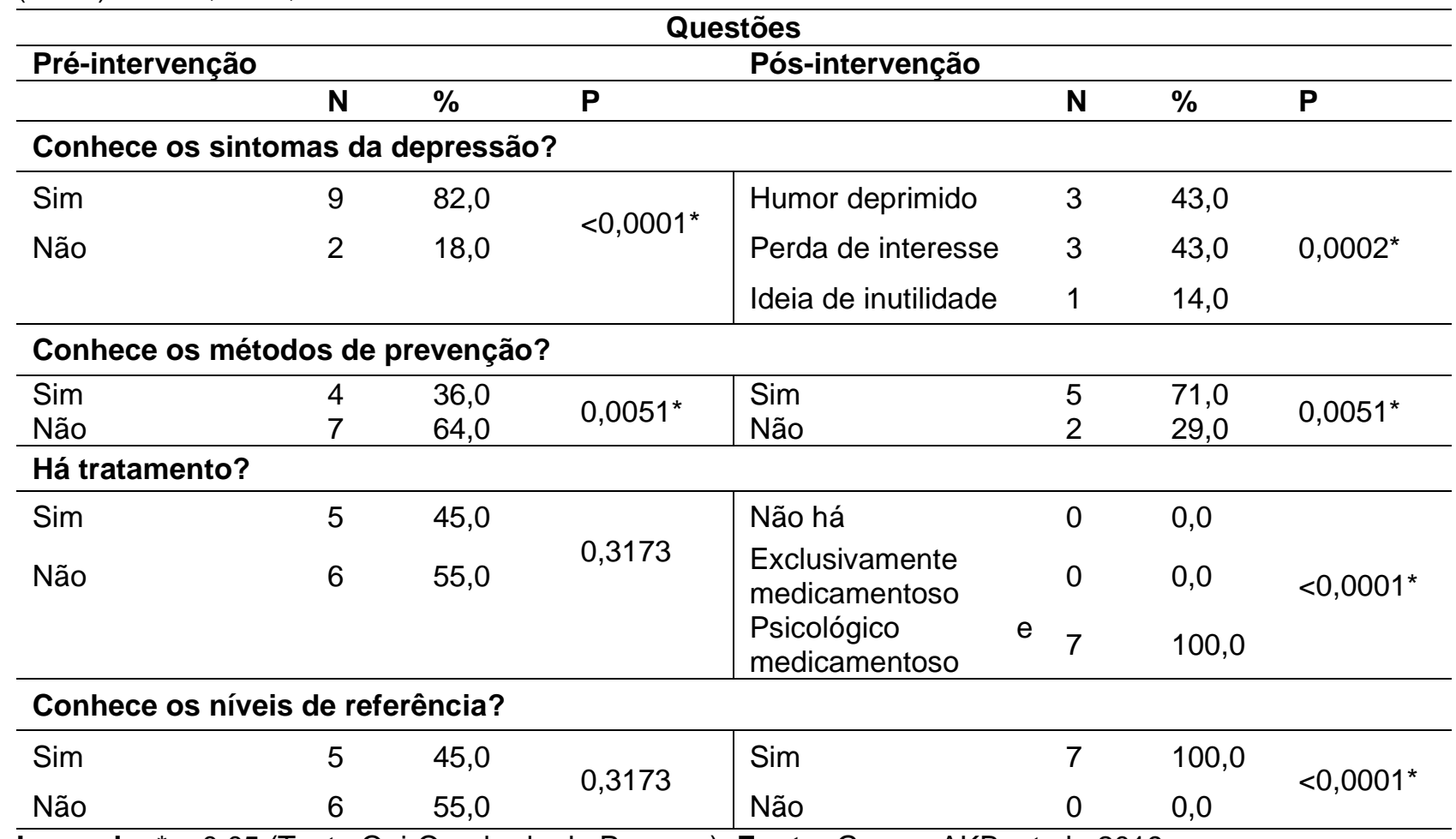

Legenda: ${ }^{*} \mathrm{p}<0,05$ (Teste Qui-Quadrado de Pearson). Fonte: Gomes AKB, et al., 2019.

\section{DISCUSSÃO}

Este estudo objetivou verificar o conhecimento dos ACS's acerca da depressão antes e após intervenção por meio de palestras socioeducativas, levando em considerando que estas são importantes ferramentas para capacitar e educar estes profissionais de saúde para detectar e atuar diante de pacientes com transtornos mentais. Pode-se perceber que, apesar de a maioria deles já terem realizado capacitações anteriores sobre o assunto, grande parte ainda apresentava déficit em relação à saúde mental, principalmente depressão, na Atenção Básica.

A área da saúde mental é complexa, possui grande intersetorialidade e transversalidade de saberes, requerendo empenho e conhecimento dos profissionais de saúde, para que possam atuar de maneira eficiente no cuidado ao paciente (OLIVEIRA EC, et al., 2017).

O ACS é fundamental para a qualidade do atendimento realizado pela equipe da ESF, inclusive no atendimento à saúde mental. Isto se deve ao fato de que o mesmo mantém contato direto com as famílias, tendo a possibilidade de encontrar usuários com problemas e encaminhá-los a ESF, sendo assim, um elo entre os usuários e a equipe de saúde (SILVA JUNIOR RF, et al., 2016).

Em relação ao tempo de atuação e formação para ACS, os resultados do presente estudo mostram que $100 \%$ dos ACS's atuam há mais de dois anos na profissão e $73 \%$ receberam formação inicial para ingressar na profissão, o que vai de encontro ao estudo de Harada OL e Soares MH (2010), que avaliaram a percepção de ACS's da cidade de Londrina - PR sobre o preparo para detecção da depressão, e verificaram que 55,6\% dos deles atuavam há menos de 15 meses na profissão e não receberam formação inicial para ACS.

No que concerne à capacitação em saúde mental, todos os participantes reconhecem a importância do conhecimento para uma boa prática profissional, afirmando ser válido o treinamento acerca do assunto. Porém, a maioria destes não apresentava o conhecimento necessário para tal atuação. Identifica-se, portanto, que a atuação dos profissionais estava prejudicada pela falta de conhecimento, apesar do empenho em ajudar a população assistida pela ESF do Canal da Visconde. 
Este resultado contraria o encontrado por Harada OL e Soares MH (2010), os quais observaram que $77,8 \%$ dos ACS's entrevistados consideravam necessário o treinamento em saúde mental e todos referiram conhecer ou já ter tido contato com pessoas com algum transtorno mental, apesar de nenhum deles ter recebido formação ou capacitação em saúde mental.

A falta de capacitação e treinamento adequado dos ACS é um fator que interfere na prestação de assistência à comunidade, pois não há como intervir sem ter conhecimento do assunto (SILVA JUNIOR RF, et al., 2016).

Dessa forma, apesar de a maioria dos participantes terem relatado já possuir capacitação sobre o assunto, a mesma pareceu não ser suficiente quando analisado o resultado do primeiro questionário. Este fato reforçou a necessidade da intervenção educativa na equipe profissional.

Contrariando estes achados, Cabral TM e Albuquerque PC (2015), em um estudo realizado na região metropolitana de Recife - PE, buscaram identificar a percepção de ACS's sobre os problemas de saúde mental na população, onde verificaram que estes possuíam pouca ou nenhuma formação, capacitação, treinamento ou preparação para intervir nas demandas de saúde mental da comunidade, no entanto reconheciam a necessidade dessa formação para compreensão da complexidade que envolve o sofrimento psíquico e a consequente melhoria de seus processos de trabalho.

Em outro estudo que avaliou o conhecimento de ACS's sobre os transtornos mentais e de comportamento em uma cidade do interior de Minas Gerais, 92,3\% deles disseram que nunca participaram de alguma capacitação ou treinamento em saúde mental, contrariando o resultado do presente estudo, em que $91 \%$ do ACS's afirmaram terem recebido capacitação em anos anteriores.

Os autores pontuam a necessidade e importância do treinamento do ACS sobre saúde mental, o que irá contribuir para a assistência aos pacientes portadores de transtornos mentais, possibilitando a identificação precoce desses transtornos e o acionamento rápido da equipe, evitando a cronificação das crises, bem como o incentivo as demais formas de tratamento complementares ao medicamentoso e a reinserção dessas pessoas ao convívio social (CARDOSO AV, et al., 2008).

Essas evidências se contrapõem às inúmeras mudanças que ocorreram no âmbito das políticas públicas em saúde mental no Brasil, como o movimento da Reforma Psiquiátrica, que exige qualificação dos profissionais de saúde, principalmente da APS, por ser a porta de entrada principal do usuário no sistema de saúde, diante do modelo de cuidado de base territorial preconizado (CABRAL TM e ALBUQUERQUE PC, 2015).

Vale ressaltar que desde de 2002, o Ministério da Saúde desenvolve o Programa Permanente de Formação de Recursos Humanos para a Reforma Psiquiátrica, que incentiva, apoia e financia a implantação de núcleos de formação em saúde mental para a rede pública, por meio de convênios estabelecidos com a participação de universidades federais, municípios e estados, inclusive com alguns cursos voltados somente para a atualização de ACS's em saúde mental realizados em Minas Gerais e Santa Catarina (BRASIL, 2007).

Estudos mostram certo desinteresse e receio de profissionais de saúde quando se trata de transtornos mentais, devido a estigmatização ainda presente na sociedade de tais pessoas serem perigosas - apesar das lutas pela desinstitucionalização e reforma psiquiátrica (SILVA JUNIOR RF, et al., 2016).

Nesse sentido, o presente trabalho buscou na sua intervenção desmistificar esse conceito errôneo sobre os portadores de transtornos mentais, objetivando maior aproximação da comunidade necessitada.

A depressão apesar de frequente, é uma doença subdiagnosticada e subtratada, sendo muitas vezes não detectada em centros de cuidado primário (WENCESLAU LD e ORTEGA F, 2015). Em acordo com este dado, foi analisado que apesar da maioria dos participantes do estudo saberem da frequência da doença, não sabiam identificar fatores importantes, como o seu curso clínico crônico e a mesma ser incapacitante.

Colaborando com o subdiagnóstico e subtratamento, estudos mostram que apenas casos mais graves recebem atenção especializada em saúde mental, fato que pode ser reforçado pela falta de instrução anteriormente referida (SILVA JUNIOR RF, et al., 2016; CUIJPERS P, et al., 2012). 
A gravidade do quadro de depressão pode ser aumentada pela demora da identificação do problema, bem como do atendimento profissional. Dessa forma, é imprescindível saber como funciona o atendimento de pessoas com transtorno mental e os níveis de referência em saúde mental (CUIJPERS P, et al., 2012). Mais uma vez a maioria dos participantes do estudo demonstraram não possuir entendimento do assunto, fato abordado na intervenção.

Prevenção compreende qualquer intervenção que visa prevenir o aparecimento de novos casos de transtornos mentais em pessoas que ainda não preenchem critérios para tal transtorno. Estudos demonstraram que ações preventivas podem reduzir a incidência de novos episódios de transtorno depressivo maior em cerca de $25 \%$ dos pacientes (CUIJPERS P, et al., 2012). Desse modo, constatando que grande parte dos ACS's questionados na pesquisa não sabiam prevenir a depressão, medidas preventivas foram ensinadas na intervenção educativa com sucesso, notado após respostas no segundo questionário.

É importante citar também sobre o planejamento em saúde voltado à participação social dos ACS, referente às ações mínimas a serem desenvolvidas e gestão de saúde mental a qual inclui, o diagnóstico, o plano de ação que visa estabelecer metas, estratégias, obtenção de recursos e o cronograma, a execução, o acompanhamento e avaliação do indivíduo portador de depressão e a capacitação destes ACS para identificar danos e agravos (SOUZA LGS, et al., 2012).

Identificamos que dentre a totalidade dos profissionais pesquisados, $27 \%$ trabalha na estratégia saúde da família por um período superior a 24 meses, sem possuir formação para atuar como ACS. Dentro do modelo da atenção básica, este dado reflete um déficit em gestão em saúde quando comparado à necessidade da população ao atendimento em saúde mental, apresentado, desta forma, um percentual de profissionais sem capacitação para atuar no modelo assistencial de saúde mental.

\section{CONCLUSÃO}

A pesquisa demonstrou que os profissionais conhecem a importância do assunto, e que uma intervenção precoce pode ser fundamental na vida de qualquer paciente. Por outro lado, a maioria desconhecia fatores como curso clínico da doença, sua prevenção e o fato de ser uma condição com potencial incapacitante. Por estes motivos, o presente trabalho pretende contribuir com o conhecimento dos agentes comunitários de saúde, além de proporcionar uma maior diversificação para a literatura do tema, auxiliando em futuros estudos e buscando maior envolvimento com a depressão, assunto de extrema relevância para a saúde pública.

\section{REFERÊNCIAS}

1. BRASIL. Ministério da Saúde. Organização Pan-Americana de Saúde. Universidade Estadual de Campinas. Prevenção do suicídio: manual dirigido a profissionais das equipes de saúde mental. Brasília: MS/OPAS/UNICAMP; 2006; 76p.

2. BRASIL. Ministério da Saúde. Secretaria de Atenção à Saúde/DAPE. Saúde Mental no SUS: acesso ao tratamento e mudança do modelo de atenção. Relatório de Gestão 2003-2006. Brasília: Ministério da Saúde; 2007; 85p.

3. BRASIL. Ministério da Saúde. Secretaria de Atenção à Saúde. Departamento de Atenção Básica. Política Nacional de Atenção Básica. Brasília: Ministério da Saúde; 2012; 110 p.

4. CABRAL TMN, ALBUQUERQUE PC. Saúde mental sob a ótica de Agentes Comunitários de Saúde: a percepção de quem cuida. Saúde debate, 2015; 39(104):159-171.

5. CARDOSO AVM, et al. Conhecimento dos agentes comunitários de saúde sobre transtorno mental e de comportamento, em uma cidade de Minas Gerais. Cogitare Enferm, 2008; 13(2):235-243.

6. CUIJPERS P, et al. Preventing depression: a global priority. JAMA, 2012; 307(10):1033-1034.

7. FLECK MP, et al. Revisão das diretrizes da Associação Médica Brasileira para o tratamento da depressão (Versão integral). Revista Brasileira de Psiquiatria, 2009; 31(Supl I):S7-17.

8. HARADA OL, SOARES MH. A percepção do agente comunitário de saúde para identificar a depressão. SMAD, Rev Eletrônica Saúde Mental Álcool Drog, 2010; 6(2): 315-336.

9. KODA MY, FERNANDES MIA. A reforma psiquiátrica e a constituição de práticas substitutivas em saúde mental: uma leitura institucional sobre a experiência de um núcleo de atenção psicossocial. Cadernos de Saúde Pública, 2007; 23(6):1455-1461.

10. OLIVEIRA EC, et al. O cuidado em saúde mental no território: concepções de profissionais da atenção básica. Escola Anna Nery, 2017; 21(3):e20160040. 
11. PILANIA M, et al. Prevalence of depression among the elderly (60 years and above) population in India, 1997-2016: a systematic review and meta-analysis. BMC Public Health, 2019; 19:832.

12. RAIC M. Depression and heart diseases: leading health problems. Medicina Academica Mostariensia, 2017; 5(12):52-59.

13. REFORMA PSIQUIÁTRICA: excluir não. 2016. In: CENTRO CULTURAL do Ministério da Saúde. Brasília: Ministério da Saúde. Disponível em: http://www.ccs.saude.gov.br/mem'oria\%20da\%loucura/mostra/excluir.html. Acesso em: 26 set. 2016.

14. SILVA JUNIOR RF, et al. O papel do agente comunitário de saúde no atendimento ao portador de transtorno mental. REAS, Revista Eletrônica Acervo Saúde, 2016; 8(1):841-845.

15. SOUZA LGS, et al. Saúde Mental na Estratégia Saúde da Família: revisão da literatura brasileira. Saúde Soc, 2012; 21(4):1022-1034.

16. VITTA FCF, et al. O Transtorno mental na percepção de Agentes Comunitários de Saúde na Estratégia Saúde da Família. Saúde em Debate, 2012; 3(93):225-233.

17. WENCESLAU LD, ORTEGA F. Saúde mental na atenção primária e Saúde Mental Global: perspectivas internacionais e cenário brasileiro. Interface. Botucatu, 2015; 19(55):1121-1132.

18. WORLD HEALTH ORGANIZATION. Depression and other common mental disorders: global health estimates. Geneva: WHO; 2017; 24p. 